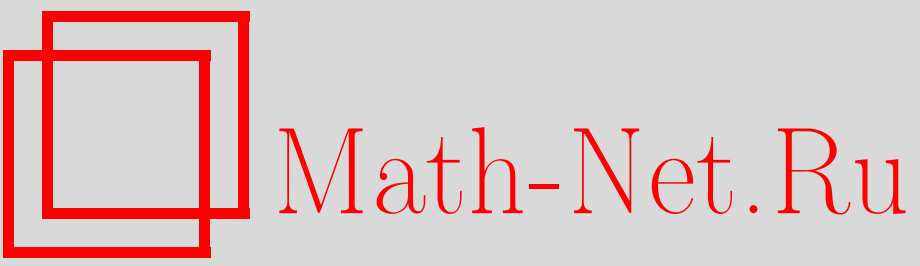

С. М. Ташпулатов, О спектрах и локализованных примесных состояниях одномагнонных систем в изотропной примесной ферромагнитной модели Гейзенберга, ТMФ, 2001, том 126, номер 3, 482-488

DOI: https://doi.org/10.4213/tmf443

Использование Общероссийского математического портала Math-Net.Ru подразумевает, что вы прочитали и согласны с пользовательским соглашением

http: //www.mathnet.ru/rus/agreement

Параметры загрузки:

IP: 54.162 .85 .209

26 апреля 2023 г., 12:21:11 


\section{О СПЕКТРАХ И ЛОКАЛИЗОВАННЫХ ПРИМЕСНЫХ СОСТОЯНИЯХ ОДНОМАГНОННЫХ СИСТЕМ В ИЗОТРОПНОЙ ПРИМЕСНОЙ ФЕРРОМАГНИТНОЙ МОДЕЛИ ГЕЙЗЕНБЕРГА}

Рассматривается одномагнонная система в изотропной примесной модели Гейзенберга. Исследуются спектр и локализованные примесные состояния этой системы на $\nu$-мерной целочисленной решетке $Z^{\nu}$. Показано, что система имеет не более трех локализованных примесных состояний (без учета кратности вырождений их энергии). Найдены области существования этих состояний и вычислены кратности вырождений их энергии.

Интенсивное развитие физики пленочного состояния, а также применение пленок в различных областях физики и техники обусловливают значительный интерес к изучению локализованных примесных состояний (ЛПС) магнетиков.

ЛПС в ферромагнетике Гейзенберга с ферромагнитной или антиферромагнитной примесями изучались в работах [1]-[6], где подробно рассматривались случаи линейной или кубической решетки. Было показано, что в линейном случае сушествует два типа ЛПС, а в случае кубической решетки - три. Насколько нам известно, случай плоской решетки подробно не исследован.

В данной работе изучается одномагнонная система в примесной модели Гейзенберга на $\nu$-мерной решетке $Z^{\nu}$. Исследуются спектр и ЛПС этой системы. Рассматривается как случай ферромагнетика с ферромагнитной примесью, так и случай ферромагнетика с антиферромагнитной примесью. Показывается, что в $\nu$-мерном случае система имеет не более трех (без учета кратности вырождений их энергии) ЛПС, и находятся условия существования этих состояний. Определяется местоположение их энергии относительно непрерывного спектра системы.

Результаты работ [1]-[6] могут быть получены из наших как частные случаи.

Отметим, что применяемые далее методы исследования отличаются от методов работ [1]-[6] простотой и полнотой полученных результатов. В отличие от [1]-[6] явно показано, что при изменении значений параметров билинейного обменного взаимодействия меняется количество ЛПС системы.

${ }^{*}$ Институт ядерной физики Академии наук Республики Узбекистан, Ташкент, пос. Улугбек, Республика Узбекистан 
Гамильтониан рассматриваемой системы имеет вид

$$
H=J \sum_{m, \tau}\left(S_{m} S_{m+\tau}\right)+\left(J_{0}-J\right) \sum_{\tau}\left(S_{0} S_{\tau}\right)
$$

где $J$ и $J_{0}$ - параметры билинейного обменного взаимодействия между атомами и между атомами и примесями, соотвественно, $S_{m}=\left(S_{m}^{x} ; S_{m}^{y} ; S_{m}^{z}\right)$ - оператор атомного спина величины $s=1 / 2$ в узле $m$, а по $\tau$ ведется суммирование по ближайшим соседям. Положим $S_{m}^{ \pm}=S_{m}^{x} \pm i S_{m}^{y}$, где $S_{m}^{+}$и $S_{m}^{-}$- соответственно операторы рождения и уничтожения магнона в узле $m$.

Гамильтониан (1) действует в симметрическом фоковском пространстве $\mathcal{H}$. Обозначим через $\varphi_{0}$ вектор, называемый вакуумным и однозначно определяемый условиями $\mathcal{S}_{m}^{+} \varphi_{0}=0, \mathcal{S}_{m}^{z} \varphi_{0}=\varphi_{0} / 2$, где $\left\|\varphi_{0}\right\|=1$. Вектор $\mathcal{S}_{m}^{-} \varphi_{0}$ описывает состояние системы одного магнона, находяшегося в узле $m$, со значением спина $s=1 / 2$. Векторы $\left\{S_{m}^{-} \varphi_{0}\right\}$ образуют ортонормальную систему. Пространство, натянутое на эти векторы, обозначим через $\widehat{\mathcal{H}}_{1}$. Это пространство евклидово относительно естественного скалярного произведения. Замыкание этого пространства обозначим через $\mathcal{H}_{1}$. Оно называется пространством одномагнонных состояний.

ПРЕДЛОЖЕНИЕ 1. Пространство $\mathcal{H}_{1}$ инвариантно относительно оператора $H$. Oператор $H_{1}=\left.H\right|_{\mathcal{H}_{1}}$ является ограниченным самосопряженным оператором, который порождает ограниченныц самосопряжсеннй оператор $\bar{H}_{1}$, действующий в пространстве $l_{2}\left(Z^{\nu}\right)$ по формуле

$$
\begin{aligned}
\left(\bar{H}_{1} f\right)(p)=\sum_{\tau} & {\left[-J f(p)+\frac{J}{2}(f(p+\tau)+f(p-\tau))\right]+} \\
& +\left(J_{0}-J\right) \sum_{\tau}\left[\left(-\delta_{p, 0}-\delta_{p, \tau}\right) f(p)+\left(\delta_{p, \tau} f(0)+\delta_{p, 0} f(\tau)\right)\right]
\end{aligned}
$$

где $\delta_{k, j}$ - символ Кронекера. Сам оператор $H_{1}$ действует на вектор $\Psi \in \mathcal{H}_{1}$ по формуле

$$
H_{1} \Psi=\sum_{p}\left(\bar{H}_{1} f\right)(p) S_{p}^{-} \varphi_{0} .
$$

Предложение 1 доказывается с использованием известных коммутационных соотношений между операторами $S_{m}^{+}, S_{p}^{-}$и $S_{q}^{z}$.

Используя критерий Вейля [7], можно доказать, что спектры операторов $H_{1}$ и $\bar{H}_{1}$ совпадают.

Наша цель состоит в изучении спектра оператора $H_{1}$. Это удобно сделать в квазиимпульсном представлении. Обозначим через $\mathcal{F}$ преобразование Фурье

$$
\mathcal{F}: l_{2}\left(Z^{\nu}\right) \rightarrow L_{2}\left(T^{\nu}\right)
$$

Здесь $T^{\nu}-\nu$-мерный тор, снабженньй нормированной мерой Лебега $d \lambda: \lambda\left(T^{\nu}\right)=1$. 
ПРЕДЛОЖЕНИЕ 2. Пусть $\mathcal{F}: \mathcal{H}_{1} \rightarrow \widetilde{\mathcal{H}}_{1} \equiv L_{2}\left(T^{\nu}\right)$ - преобразование Фурье. Оператор $\bar{H}_{1}$ переходит в оператор $\widetilde{H}_{1}=\mathcal{F} \bar{H}_{1} \mathcal{F}^{-1}$, который действует в пространстве $L_{2}\left(T^{\nu}\right)$ по формуле

$$
\left(\widetilde{H}_{1} f\right)(x)=h(x) f(x)+\int_{T^{\nu}} h_{1}(x ; t) f(t) d t
$$

$2 \partial e$

$$
\begin{gathered}
h(x)=-2 J \nu+2 J \sum_{i=1}^{\nu} \cos x_{i} \\
h_{1}(x ; t)=-2 \varepsilon\left\{\nu+\sum_{i=1}^{\nu}\left[\cos \left(x_{i}-t_{i}\right)-\cos x_{i}-\cos t_{i}\right]\right\}
\end{gathered}
$$

здесъ $\varepsilon=J_{0}-J ; \quad x, t \in T^{\nu}$.

Доказательство предложения 2 проводится непосредственно с использованием преобразования Фурье.

Известно, что непрерывный спектр оператора $\widetilde{H}_{1}$ не зависит от функции $h_{1}(x ; t)$ и заполняет весь отрезок $\left[m_{\nu}, \mathcal{M}_{\nu}\right]$, где $m_{\nu}=\min _{x \in T^{\nu}} h(x), \mathcal{M}_{\nu}=\max _{x \in T^{\nu}} h(x)$.

Собственная функция $\varphi \in L_{2}\left(T^{\nu}\right)$ оператора $\widetilde{H}_{1}$, отвечаюшая собственному значению $z$, называется ЛПС оператора $\widetilde{H}_{1}$, а величина $z$ - энергией этого состояния.

Рассмотрим оператор $K_{\nu}(z)$, действующий в пространстве $\widetilde{H}_{1}$ по формуле

$$
\left(K_{\nu}(z) f\right)(x)=\int_{T^{\nu}} \frac{h_{1}(x ; t)}{h(t)-z} f(t) d t, \quad x, t \in T^{\nu} .
$$

Он является компактным оператором в пространстве $L_{2}\left(T^{\nu}\right)$ для значений $z \notin$ $\left[m_{\nu}, \mathcal{M}_{\nu}\right]$. Обозначим через $\Delta_{\nu}(z)$ определитель Фредгольма оператора $E+K_{\nu}(z)$, где $E$ - единичньй оператор в пространстве $\widetilde{\mathcal{H}_{1}}$.

ЛЕмма 1. Определитель Фредгольма $\Delta_{\nu}(z)$ оператора $E+K_{\nu}(z)$ имеет вид

$$
\Delta_{\nu}(z)=\left(1-2 \varepsilon J_{\nu 1}(z)\right)\left(1-2 \varepsilon J_{\nu 2}(z)\right)^{\nu}\left(1-\varepsilon J_{\nu 3}(z)\right)^{\nu-1}
$$

$2 \partial e$

$$
\begin{aligned}
& J_{\nu 1}(z)=\int_{T^{\nu}} \frac{\left(1-\cos s_{1}\right)\left(\nu-\sum_{i=1}^{\nu} \cos s_{i}\right) d s_{1} d s_{2} \ldots d s_{\nu}}{h\left(s_{1} ; s_{2} ; \ldots ; s_{\nu}\right)-z} \\
& J_{\nu 2}(z)=\int_{T^{\nu}} \frac{\sin ^{2} s_{1} d s_{1} d s_{2} \ldots d s_{\nu}}{h\left(s_{1} ; s_{2} ; \ldots ; s_{\nu}\right)-z} \\
& J_{\nu 3}(z)=\int_{T^{\nu}} \frac{\left(\cos s_{1}-\cos s_{2}\right)^{2} d s_{1} d s_{2} \ldots d s_{\nu}}{h\left(s_{1} ; s_{2} ; \ldots ; s_{\nu}\right)-z}
\end{aligned}
$$

Доказательство леммы 1 получается непосредственно с использованием вида функций $h(x)$ и $h_{1}(x ; t)$. 
Лемма 2. Число $z=z_{0} \notin\left[m_{\nu}, \mathcal{M}_{\nu}\right]$ является собственныцм значением оператора $\widetilde{H}_{1}$ тогда и только тогда, когда оно является нулем функции $\Delta_{\nu}(z)$, т.е. $\Delta_{\nu}(z)=0$.

ДоКАЗАТЕЛЬСТво. Пусть $z=z_{0}$ - собственное значение оператора $\widetilde{H}_{1}$. Это означает, что число $\mu=-1$ является собственным значением оператора $K_{\nu}\left(z_{0}\right)$, т.е. однородное уравнение

$$
f(x)+\int_{T^{\nu}} \frac{h_{1}(x ; t)}{h(t)-z} f(t) d t=0
$$

имеет нетривиальное решение. Следовательно, в точке $z=z_{0}$ функция $\Delta_{\nu}(z)$ равна нулю, $\Delta_{\nu}\left(z_{0}\right)=0$.

Пусть теперь точка $z=z_{0}$ является нулем функции $\Delta_{\nu}(z): \Delta_{\nu}\left(z_{0}\right)=0$. Тогда указанное однородное уравнение также имеет нетривиальное решение. Отсюда следует, что число $z=z_{0}$ является собственным значением оператора $\widetilde{H}_{1}$.

Обозначим через $P$ пару $(J ; \varepsilon): P=(J ; \varepsilon)$ и при $\nu=1$ введем следуюшие множества значений этих пар:

$$
\begin{array}{lll}
G_{1}=\{P: J<0,0<\varepsilon \leqslant-J\}, & G_{2}=\{P: J>0,-J \leqslant \varepsilon<0\}, \\
G_{3}=\{P: J>0,0<\varepsilon \leqslant J\}, & G_{4}=\{P: J<0, J \leqslant \varepsilon<0\}, \\
G_{5}=\{P: J>0, \varepsilon>J\}, & G_{6}=\{P: J<0, \varepsilon>-J\}, \\
G_{7}=\{P: J>0, \varepsilon<-J\}, & G_{8}=\{P: J<0, \varepsilon<J\} .
\end{array}
$$

Положим $z_{1}=(J+\varepsilon)(J-2 \varepsilon-\sqrt{D}) /(2 \varepsilon), z_{2}=-(J+\varepsilon)^{2} / \varepsilon, z_{3}=(J+\varepsilon)(J-3 \varepsilon+$ $\sqrt{D}) /(2 \varepsilon)$, где $D=(J+\varepsilon)(J+9 \varepsilon)$.

Следуюшая теорема описывает изменение энергетического спектра оператора $\widetilde{H}_{1}$ в одномерном случае.

Teоpema 1. А. Если $P \in G_{1} \cup G_{2}$, mo оператор $\widetilde{H}_{1}$ не имеeт ЛПC.

Б. Если $P \in G_{3} \quad\left(P \in G_{4}\right)$, то оператор $\widetilde{H}_{1}$ имеет единственное ЛПС $\varphi$ со значением әнергии $z=z_{1}\left(z=z_{3}\right)$. Кроме того, $z_{1}<m_{1}\left(z_{3}>\mathcal{M}_{1}\right)$ и ЛПС невырожденно.

В. Если $P \in G_{5} \cup G_{6}\left(P \in G_{7} \cup G_{8}\right)$, то оператор $\widetilde{H}_{1}$ имеет ровно два ЛПС $\varphi_{1}$ и $\varphi_{2}$ со значениями әнергий, соответственно равными $z=z_{1} u z=z_{2} \quad\left(z=z_{2} u\right.$ $\left.z=z_{3}\right)$. Причем $z_{1}<z_{2}\left(z_{2}<z_{3}\right) u z_{i}<m_{1}, i=1,2\left(z_{j}>\mathcal{M}_{1}, j=2,3\right)$, и уровни әтих әнергий невырожденньь.

ДоКАЗАТЕЛЬСТво. Из формулы (5) при $\nu=1$ следует, что интегралы $J_{1 k}(z), k=$ 1,2 , вычисляются в квадратурах, а третья скобка в (5) равна единице. Вычисляя значения этих интегралов для значений $z \notin\left[m_{1}, \mathcal{M}_{1}\right]$, из уравнения $\Delta_{1}(z)=0$ для определения $z$ получаем следуюшие уравнения:

а) при $z<m_{1}$ :

$$
\begin{aligned}
& B(J, \varepsilon, z)=K(J, \varepsilon, z), \\
& C(J, \varepsilon, z)=N(J, \varepsilon, z) ;
\end{aligned}
$$


б) при $z>\mathcal{M}_{1}$ :

$$
\begin{aligned}
& B(J, \varepsilon, z)=-K(J, \varepsilon, z), \\
& C(J, \varepsilon, z)=-N(J, \varepsilon, z),
\end{aligned}
$$

где

$$
\begin{aligned}
& B(J, \varepsilon, z)=\varepsilon(2 J+z)+2 J^{2}, \\
& K(J, \varepsilon, z)=-\varepsilon \sqrt{(-2 J-z)^{2}-4 J^{2}}, \\
& N(J, \varepsilon, z)=\varepsilon z^{2}, \\
& C(J, \varepsilon, z)=-\left(\varepsilon z+2 J \varepsilon+2 J^{2}\right) \sqrt{(-2 J-z)^{2}-4 J^{2}} .
\end{aligned}
$$

Решения уравнений (6) и (7) (уравнений (8) и (9)) удовлетворяют требованиям теоремы 1.

В случае размерности $\nu=2$ введем следуюшие множества значений пар $P$ :

$$
\begin{array}{rlrl}
Q_{1} & =\{P: J<0,0<\varepsilon \leqslant-J\}, & Q_{2} & =\left\{P: J<0,-J<\varepsilon \leqslant-\frac{100}{27} J\right\} \\
Q_{3} & =\left\{P: J<0,-\frac{100}{27} J<\varepsilon \leqslant-\frac{25}{9} J\right\}, & Q_{4}=\left\{P: J<0, \varepsilon>-\frac{25}{9} J\right\}, \\
Q_{5} & =\left\{P: J<0, \frac{25}{9} J<\varepsilon<0\right\}, & Q_{6} & =\left\{P: J<0, \frac{100}{27} J<\varepsilon \leqslant \frac{25}{9} J\right\}, \\
Q_{7} & =\left\{P: J<0, \varepsilon \leqslant \frac{100}{27} J\right\}, & Q_{8} & =\left\{P: J>0,0<\varepsilon \leqslant \frac{25}{9} J\right\}, \\
Q_{9} & =\left\{P: J>0, \frac{25}{9} J<\varepsilon \leqslant \frac{100}{27} J\right\}, & Q_{10} & =\left\{P: J>0, \varepsilon>\frac{100}{27} J\right\} \\
Q_{11} & =\{P: J>0,-J \leqslant \varepsilon<0\}, & Q_{12} & =\left\{P: J>0,-\frac{25}{9} J \leqslant \varepsilon<-J\right\}, \\
Q_{13} & =\left\{P: J>0,-\frac{100}{27} J \leqslant \varepsilon<-\frac{25}{9} J\right\}, & Q_{14} & =\left\{P: J>0, \varepsilon<-\frac{100}{27} J\right\} .
\end{array}
$$

Следуюшая теорема описывает изменение энергетического спектра оператора $\widetilde{H}_{1}$ в двумерном случае.

Tеорема 2. А. Если $P \in Q_{1} \cup Q_{11}$, mо оператор $\widetilde{H}_{1}$ не имеет ЛПC.

Б. Если $P \in Q_{2} \cup Q_{8} \quad\left(P \in Q_{5} \cup Q_{12}\right)$, то оператор $\widetilde{H}_{1}$ имеет единственное ЛПС $\varphi$ со значением әнергии $z_{1}\left(z_{2}\right)$, причем $z_{1}<m_{2} \quad\left(z_{2}>\mathcal{M}_{2}\right)$. Уровень әтой энергии невырожден.

В. Если $P \in Q_{3} \cup Q_{9} \quad\left(P \in Q_{6} \cup Q_{13}\right)$, то оператор $\widetilde{H}_{1}$ имеет ровно два ЛПС $\varphi_{1}$ и $\varphi_{2}$ со значениями әнергии $z_{1}$ и $z_{2}, z_{i}<m_{2}, i=1,2\left(z_{3} u z_{4}, z_{j}>\mathcal{M}_{2}, j=3,4\right)$, и уровни этих энергий невырожденны.

Г. Если $P \in Q_{4} \cup Q_{10} \quad\left(P \in Q_{7} \cup Q_{14}\right)$, то оператор $\widetilde{H}_{1}$ имеет ровно три ЛПС $\varphi_{1}, \varphi_{2}$ и $\varphi_{3}$ со значениями энергии, соответственно равными $z_{1}, z_{2}, z_{3}\left(z_{4}, z_{5}, z_{6}\right)$, причем $z_{i}<m_{2}, \quad i=1,2,3 \quad\left(z_{j}>\mathcal{M}_{2}, j=4,5,6\right)$. Состояния $z_{1} \quad$ и $z_{3} \quad\left(\begin{array}{lll}z_{4} & u & z_{6}\end{array}\right)$ невырожденны, а состояние $z_{2}\left(z_{5}\right)$ двукратно вырожденно. 
ДокАЗАТЕЛЬСТво. Функции $J_{2 k}(z), k=1,2,3$, при $z \notin\left[m_{2}, \mathcal{M}_{2}\right]$ являются монотонными. При $z<m_{2}$ и $J<0$ функция $J_{21}(z)$ возрастает от 0 до $-(1 / 2 J)$, функция $J_{22}(z)$ возрастает от 0 до $-(9 / 50 J)$, а функция $J_{23}(z)$ возрастает от 0 до $-(27 / 100 J)$. А при $z>\mathcal{M}_{2}$ и $J<0$ эти функции возрастают соответственно от $-\infty$ до 0 , от $9 / 50 J$ до 0 и от $27 / 100 J$ до 0 . В этом легко можно убедиться, вычисляя значения интегралов $J_{2 k}\left(m_{2}\right)$ и $J_{2 k}\left(\mathcal{M}_{2}\right), k=1,2,3$. Учитьвая, что в рассматриваемом случае мера нормированная, получаем значения интегралов $J_{2 k}\left(m_{2}\right)$ и $J_{2 k}\left(\mathcal{M}_{2}\right), k=1,2,3$. В случае $J>0$ и $z<m_{2}$ функции $J_{2 k}(z), k=1,2,3$, возрастают от 0 до $\infty$, от 0 до $9 / 50 J$ и от 0 до $27 / 100 J$, соответственно. При $J>0$ и $z>\mathcal{M}_{2}$ эти функции становятся опять возрастающими. Они возрастают от $-(1 / 2 J)$ до 0 , от $-(9 / 50 J)$ до 0 и от $-(27 / 100 J)$ до 0 , соответственно. Теперь исследуя уравнение $\Delta_{2}(z)=0$, немедленно получаем доказательство теоремы 2.

В случае $\nu=3$ введем следуюшие обозначения:

$$
\begin{aligned}
a & =\int_{T^{3}} \frac{\sin ^{2} s_{1} d s_{1} d s_{2} d s_{3}}{3-\cos s_{1}-\cos s_{2}-\cos s_{3}}=\int_{T^{3}} \frac{\sin ^{2} s_{1} d s_{1} d s_{2} d s_{3}}{3+\cos s_{1}+\cos s_{2}+\cos s_{3}}, \\
b & =\int_{T^{3}} \frac{\left(\cos s_{1}-\cos s_{2}\right)^{2} d s_{1} d s_{2} d s_{3}}{3-\cos s_{1}-\cos s_{2}-\cos s_{3}}=\int_{T^{3}} \frac{\left(\cos s_{1}-\cos s_{2}\right)^{2} d s_{1} d s_{2} d s_{3}}{3+\cos s_{1}+\cos s_{2}+\cos s_{3}} .
\end{aligned}
$$

Ясно, что $0<a<b<1$ и $2 a<b$. Теперь введем следуюшие множества пар $P$ :

$$
\begin{aligned}
Q_{1} & =\{P: J<0,0<\varepsilon<-J\}, & Q_{2} & =\left\{P: \frac{J}{3}<\varepsilon<0, J<0\right\}, \\
Q_{3} & =\left\{P: J>0,0<\varepsilon<\frac{J}{3}\right\}, & Q_{4} & =\{P: J>0,-J<\varepsilon<0\}, \\
Q_{5} & =\left\{P: J<0,-J \leqslant \varepsilon<-\frac{2 J}{b}\right\}, & Q_{6} & =\left\{P: J>0, \frac{J}{3} \leqslant \varepsilon<\frac{2 J}{b}\right\}, \\
Q_{7} & =\left\{P: J<0, \frac{2 J}{b}<\varepsilon \leqslant \frac{J}{3}\right\}, & Q_{8} & =\left\{P: J>0,-\frac{2 J}{b}<\varepsilon \leqslant-\frac{J}{a}\right\} \\
Q_{9} & =\left\{P: J<0,-\frac{2 J}{b} \leqslant \varepsilon<-\frac{J}{a}\right\}, & Q_{10} & =\left\{P: J>0,-\frac{2 J}{b} \leqslant \varepsilon<\frac{J}{a}\right\} \\
Q_{11} & =\left\{P: J<0, \frac{J}{a}<\varepsilon \leqslant \frac{2 J}{b}\right\}, & Q_{12} & =\left\{P: J>0,-\frac{J}{a}<\varepsilon \leqslant-\frac{2 J}{b}\right\} \\
Q_{13} & =\left\{P: J<0, \varepsilon \geqslant-\frac{J}{a}\right\}, & Q_{14} & =\left\{P: J>0, \varepsilon \geqslant \frac{J}{a}\right\} \\
Q_{15} & =\left\{P: J<0, \varepsilon \leqslant \frac{J}{a}\right\}, & Q_{16} & =\left\{P: J>0, \varepsilon \leqslant-\frac{J}{a}\right\}
\end{aligned}
$$

Teopema 3. А. Пусть $P \in Q_{1} \cup Q_{2} \cup Q_{3} \cup Q_{4}$. Тогда оператор $\widetilde{H}_{1}$ не имеет ЛПС.

Б. Пусть $P \in Q_{5} \cup Q_{6} \quad\left(P \in Q_{7} \cup Q_{8}\right)$. Тогда оператор $\widetilde{H}_{1}$ имеет единственное ЛПС со значением энергии $z<m_{3}\left(z>\mathcal{M}_{3}\right)$. Уровень этой энергии невырожден. 
B. Пусть $P \in Q_{9} \cup Q_{10} \quad\left(P \in Q_{11} \cup Q_{12}\right)$. Тогда оператор $\widetilde{H}_{1}$ имеет ровно два ЛПС $\varphi_{1}$ и $\varphi_{2}$ со значениями энергии, соответственно равными $z_{1}$ и $z_{2} \quad\left(\begin{array}{lll}z_{3} & z_{4}\end{array}\right)$, причем $z_{i}<m_{3}, \quad i=1,2 \quad\left(z_{j}>\mathcal{M}_{3}, j=3,4\right)$. Кроме того, состояние $z_{1} \quad\left(z_{3}\right)$ невырожденно, а состояние $z_{2}\left(z_{4}\right)$ двукратно вырожденно.

Г. Пусть $P \in Q_{13} \cup Q_{14} \quad\left(P \in Q_{15} \cup Q_{16}\right)$. Тогда оператор $\widetilde{H}_{1}$ имеет ровно три ЛПС $\varphi_{1}, \varphi_{2}$ и $\varphi_{3}$ со значениями әнергии, соответственно равньми $z_{1}, z_{2}, z_{3} \quad\left(z_{4}\right.$, $\left.z_{5}, z_{6}\right)$. Причем $z_{i}<m_{3}, \quad i=1,2,3 \quad\left(z_{j}>\mathcal{M}_{3} ; j=4,5,6\right)$. Кроме того, состояние $z_{1}$ невырожденно, $z_{2}$ двукратно вырожденно, а $z_{3}$ трехкратно вырожденно.

Теорема 3 доказывается так же, как и теорема 2. При доказательстве используются монотонность функций $J_{3 k}(z), k=1,2,3$, при $z \notin\left[m_{3}, \mathcal{M}_{3}\right]$ и значения интеграла Ватсона [8]. При этом учтено, что в рассматриваемом случае мера нормированная.

Аналогично можно доказать, что в $\nu$-мерном случае система имеет не более трех ЛПС (без учета кратности вырождений их энергий) со значениями энергий $z_{i} \notin$ $\left[m_{\nu}, \mathcal{M}_{\nu}\right], i=1,2,3$. Кроме того, уровень этих энергий соответственно невырожден, $\nu$ - и $(\nu-1)$-кратно вырожден. Так же можно определить области существования ЛПС. Следовательно, в системе могут существовать не более трех видов ЛПС.

\section{Список литературы}

[1] Ю. А. Изюмов, М. В. Медведев. ЖЭТФ. 1965. Т. 48. № 2. С. 574-586.

[2] В. В. Ганн, Л. Г. Зазунов. ФТТ. 1973. Т. 15. № 12. С. 3535-3569.

[3] Yung-Li Wang, H. Callen. Phys. Rev. 1967. V. 160. № 2. P. 358-363.

[4] T. Oguchi, I. Ono. J. Phys. Soc. Japan. 1969. V. 26. № 1. P. 32-42.

[5] T. Wolfram, J. Callaway. Phys. Rev. 1963. V. 130. № 6. P. 2207-2217.

[6] I. Ono, Y. Endo. Phys. Rev. Lett. A. 1972. V. 41. № 5. P. 440-442.

[7] М. Рид, Б. Саймон. Методы современной математической физики. Т. 1. Функциональный анализ. М.: Мир, 1977.

[8] В. В. Вальков, С.Г. Овчинников, О.П. Петраковский. ФТТ. 1988. Т. 30. № 10. C. $3044-3047$. 